\title{
Rastreio Oncológico - Porquê, Para Quem, Quando e Como
}

\author{
Vitor José Lopes Rodrigues \\ ${ }^{1}$ Centro de Investigação em Meio Ambiente, Genética e Oncobiologia (CIMAGO), Portugal.; Email: \\ vrodrigues@netcabo.pt
}

\begin{abstract}
Resumo
A patologia oncológica corresponde a uma significativa parcela da incidência e mortalidade das doenças crónicas nas sociedades mundiais, sobretudo daquelas mais desenvolvidas economicamente. A sua prevenção secundária tornou-se uma arma de reconhecida importância, nomeadamente através de âmbito populacional e de aplicação de metodologias e técnicas padronizadas de rastreio. São descritos os princípios gerais de rastreio oncológico, bem como a sua aplicação e adequação aos casos de cancro da mama feminina, colo do útero e segmento colorectal.
\end{abstract}

Palavras-chave: rastreio de cancro colorectal; rastreio de cancro do cólo do útero; rastreio de cancro de mama; rastreio oncológico.

\begin{abstract}
The oncology pathology corresponds to a significant portion of the chronic diseases incidence and mortality in the world societies, especially those that are more developed economically. The secondary prevention has become a weapon of recognized importance, namely through population-based scope and organization screening standard methods and techniques. The general principles of cancer screening, as well as its application and suitability for the female breast, cervix and colorectal cancers, are described.
\end{abstract}

Keywords: breast cancer screening; cancer screening; cervix cancer screening; colorectal cancer screening.

\section{Introdução}

As doenças oncológicas constituem um conjunto de entidades nosológicas com uma importância substantiva e progressiva no panorama da morbi-mortalidade a nível mundial. No entanto, a sua heterogeneidade, decorrente da possibilidade de eclosão em qualquer órgão ou tecido do corpo humano, influencia decisivamente as diferentes causalidades, incidências, prevalências, diagnósticos, tratamentos e mortalidades.

Sendo uma patologia intrinsecamente associada ao meio ambiente e aos comportamentos, tem uma significativa capacidade de prevenção primária (a profilaxia), através do afastamento dos factores de risco importantes, como sejam o tabagismo, a alimentação inadequada, o sedentarismo, o consumo excessivo de bebidas alcoólicas, o ambiente profissional de risco, os incorrectos comportamento sexuais e reprodutivos e algumas infecções. No entanto, essa sua capacidade encontra-se, na prática, bastante limitada, pois tais factores de risco estão amplamente associados a atitudes e comportamentos muito intrincados nas sociedades e nos seres humanos. É muito difícil induzir esse tipo de alterações comportamentais, tanto mais que a) - não existe uma causalidade inequívoca (excepto nos casos do tabagismo e da infecção por HPV), b) - é raro que um único factor de risco seja o responsável pela causalidade e c) - os tempos de latência entre a exposição e a eclosão da doença são normalmente muito dilatados. 
Outra arma, fundamental, disponível para a diminuição da mortalidade (e, em algumas localizações, da incidência) por esta patologia é a prevenção secundária. Esta define-se como a detecção e tratamento da doença antes que surjam sinais e sintomas, ou seja, na fase pré ou infra-clínica.

Há várias modalidades de prevenção secundária de cancro:
A - Diagnóstico precoce
B - Rastreio "oportunístico"
C - Rastreio organizado populacional
D - Cuidados personalizados

A - O Diagnóstico precoce tem uma base individual, pois requer que seja a pessoa a procurar os cuidados de saúde devido a um sinal ou sintoma suspeito. Mas requer, igualmente, que o indivíduo tenha a literacia em saúde necessária para identificar a sua existência e esteja predisposto a recorrer a esses cuidados de saúde, adoptando uma actuação activa de prevenção individual.

B - O Rastreio "oportunístico" assenta mais numa atitude tendencialmente populacional, embora casuística e não organizada. Assim, aquando duma visita aos cuidados de saúde, por alguma causa não relacionada com motivos de prevenção oncológica, os profissionais aproveitam esse momento para realizar, ou requisitar, testes de rastreio/diagnóstico dirigidos à deteç̧ão precoce de patologia oncológica. Tem uma reduzida eficiência populacional, conduzindo também a desigualdades sociais, pois apenas aqueles que estão inseridos activamente nossistemas de saúde beneficiam com esta metodologia. Pode, pois, considerar-se que reflecte uma insuficiência ou falha na igualdade e equidade dos sistemas de saúde, pelo que, conceptualmente, tem um interesse muito limitado e não deveria existir.

C - O Rastreio organizado populacional tem uma nítida perspectiva comunitária, dirigida para os indivíduos sem sinais e sem sintomas, e está sujeito à análise custo-efectividade (pré e pós-intervenção) e estuda a pessoa num processo multifásico com intervenções de agressividade diagnóstica progressiva.

D - Os Cuidados personalizados correspondem a uma intervenção ainda mais estruturada na procura do diagnóstico precoce em oncologia. São concebidos numa base individual através da qual qualquer pessoa deveria ter uma estratificação dos seus riscos. Assim sendo, toda a intervenção de diagnóstico precoce estaria dirigida a cada indivíduo, o que condicionaria os tipos de cancro a serem prevenidos, o tipo de diagnóstico a ser realizado, e a sua periodicidade. Trata-se de um tipo de metodologia que, a prazo, poderá ser gradualmente implementado, à medida que o conhecimento científico for avançando. Um exemplo deste tipo de actuação consiste no chamado "rastreio de populações de alto-risco", sujeitos a determinantes genéticas, hereditárias e ambientais agressivas e particulares.

A implementação do tipo de actividades de prevenção oncológica numa determinada população é condicionada pela Política de saúde que, por sua vez, se apoia em relevantes estudos provenientes da saúde pública e da economia da saúde (prioridades da sociedade, estado da situação na procura e na oferta de cuidados, caracterização da epidemiologia das referidas doenças e evidência científica).

\section{Princípios Gerais do Rastreio}

O rastreio (aqui como sinónimo de "rastreio organizado populacional") pode ser definido como a aplicação de um teste para identificar a doença assintomática e precoce, sendo o seu objectivo primário a redução da mortalidade (no caso do cancro da mama) e/ou da incidência (no caso do colo do útero e colorrectal). Além disso, um rastreio tem importantes consequências adicionais, como sejam a necessidade de se disponibilizarem vultuosos recursos financeiros, a possibilidade de haver uma potencial poupança a médio e longo prazo e, ainda, os efeitos na qualidade de vida, positivos ou negativos (Hakama et al., 2008).

Como o cancro é sempre uma doença potencialmente letal, o primeiro objectivo do rastreio oncológico (e tratamento subsequente) é o aumento da sobrevida dos doentes detectados (com a melhor qualidade de vida 
possível). O primeiro indicador da sua eficiência é a diminuição da mortalidade (específica e/ou geral), mas outros indicadores indirectos ("surrogate") podem ser usados, como a taxa de adesão da população, o estadio na detecção da doença, a menor agressividade da intervenção, o aumento da sobrevida, os indicadores de rendimento, a ocorrência de cancros de intervalo e a morbilidade psicológica. (European Commission, 2006; Arbyn et al., 2008; Bond et al., 2013)

O rastreio oncológico requer critérios a) relativos à doença (a existência de altas, incidência, prevalência e mortalidade), o conhecimento da história natural, a possibilidade de nela intervirmodificando o seu curso clínico, e, idealmente, uma significativamente grande fase pré-clínica), b) relativos ao teste de rastreio (teste simples, aceitável, com baixo custo, seguro e de aplicação rápida, bem como possuidor de adequadas sensibilidade, especificidade e valores preditivos positivo e negativo), c) relativos ao rendimento (com diagnóstico definitivo e tratamento adequado disponíveis imediatamente) e d) relativos ao efeito (com redução evidenciável e significativa da incidência e/ou, antecipados por indicadores intermédios favoráveis, semelhantes e comparáveis aos indicadores padrão). O rastreio tem também critérios de implementação e de execução bem definidos, como sejam (Wilson \& Jungner, 1968; Andermann et al., 2008):

1. O programa de rastreio deverá responder a uma necessidade reconhecida

2. Os objectivos do rastreio deverão ser definidos no início do seu planeamento

3. Deverá haver uma populacão-alvo definida

4. Deverá haver evidência científica sobre a eficiência e efectividade do programa de rastreio

5. A gestão do programa ter em atenção todos os seus componentes com uma especial atenção à coordenação dos serviços clínicos, aos procedimentos do teste e à educação permanente dos colaboradores e da população

6. Deverá haver procedimentos de controlo e garantia de qualidade, com mecanismos para minimizar os potenciais riscos do rastreio

7. O programa deverá assegurar consentimento informado nas suas diferentes fases, confidencialidade e respeito pela autonomia das pessoas rastreadas

8. O programa deverá promover a equidade e o acesso ao rastreio pela totalidade da população

9. A avaliação do rastreio deverá ser definida desde o início do seu planeamento

10. Os benefícios globais do rastreio deverão ser superiores aos malefícios

A União Europeia é taxativa na seguinte declaração, de 2003: "The screening tests ..... can only be offered on a population basis in organised screening programmes with quality assurance at all levels, if good information about benefits and risks, adequate resources for screening, follow-up with complementary diagnostic procedures and, if necessary, treatment of those with a positive screening test are available." (Council of the European Union, 2013)

O processo de rastreio, como processo multifásico, exige a organização cuidada dos seus vários componentes (figura 1 e tabela 1): 


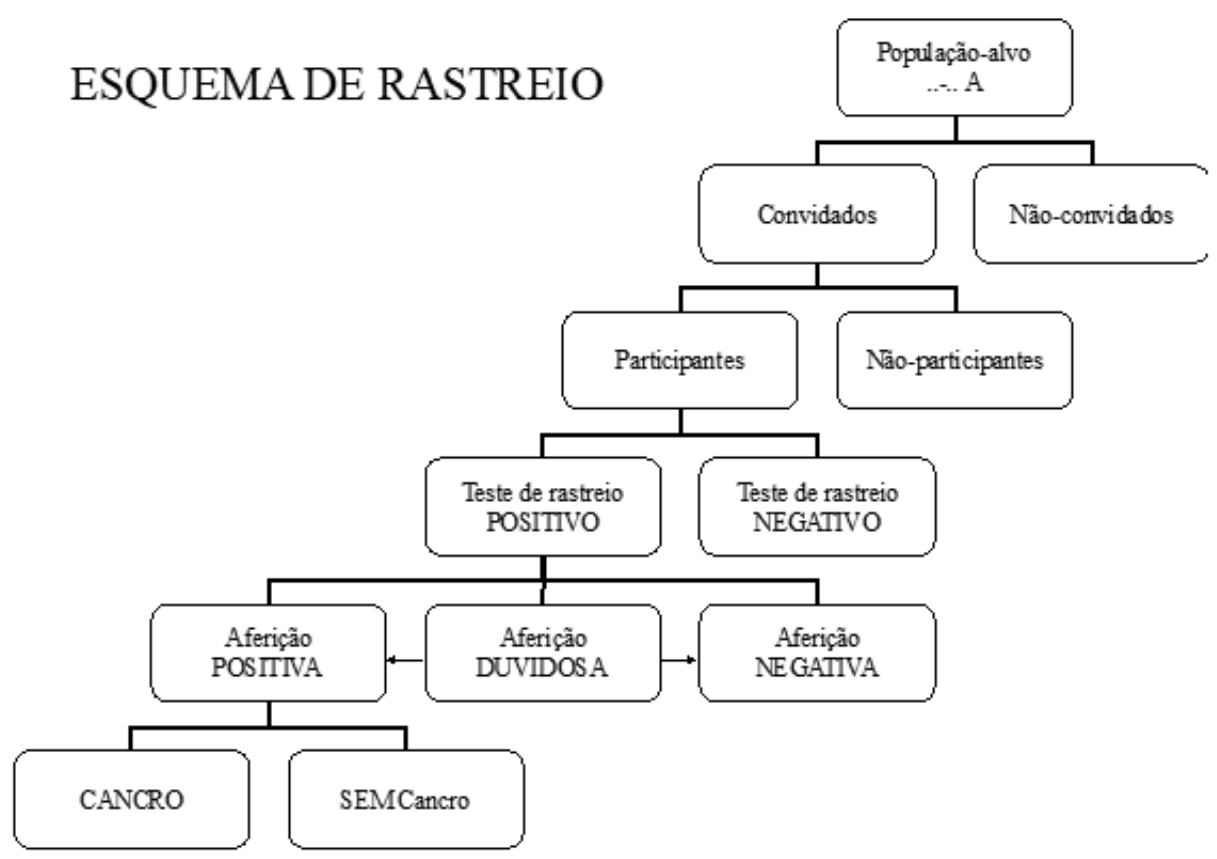

Figura 1. Processo multifásico de rastreio

Tabela 1. Componentes dos programas de rastreio oncológico

\begin{tabular}{|c|c|}
\hline Componente & Procedimentos \\
\hline Populacional & Definição da populacão-alvo \\
\hline Identificação dos indivíduos & Medidas para conseguir boas coberturas e participação \\
\hline Execução do teste & $\begin{array}{c}\text { Infra-estruturas para colher o material e a análise do material de rastreio. } \\
\text { Programas de controlo de qualidade para a obtenção do material de rastreio } \\
\text { e sua análise e interpretação. }\end{array}$ \\
\hline Clínico & $\begin{array}{l}\text { Infra-estruturas para diagnóstico, tratamento e acompanhamento dos } \\
\text { doentes com cancros (ou lesões pré-neoplásicas detectadas no rastreio). }\end{array}$ \\
\hline Coordenação & $\begin{array}{l}\text { Um sistema de referência ligando o rastreio, a unidade de rastreio e a infra- } \\
\text { estrutura clínica. } \\
\text { Um sistema que assegure o processo de convocação e re-convocação. } \\
\text { Um sistema que forneça informação de retorno aos utentes e aos } \\
\text { profissionais de saúde. }\end{array}$ \\
\hline
\end{tabular}

Adaptado de Hakama (2008)

O processo de rastreio é semelhante para todas as localizações tumorais dele passíveis. Inicia-se na identificação e individualização da população-alvo (género, grupo etário, critérios de exclusão e de inclusão), prossegue com o convite individual e personalizada, a execução e análise do teste de rastreio, a eventual convocatória para consulta clínica (de "aferição") e o eventual encaminhamento dos casos suspeitos para uma instituição hospitalar para diagnóstico final, tratamento e acompanhamento. Todo este processo deverá estar sujeito a mecanismos de controlo e garantia de qualidade, de modo a ser possível maximizar os benefícios e minimizar os malefícios.

\section{Recomendações metodológicas}

A União Europeia recomenda o rastreio organizado populacional em três localizações tumorais: mama feminina, colo do útero e colorrectal (tabela 2). 
Tabela 2. Teste primário, idade e intervalo entre testes para rastreio da mama, colo do útero e colorrectal em programas europeus organizados (Armaroli et al. 2015)

\begin{tabular}{|c|c|}
\hline Localização & \\
\hline Mama & $\begin{array}{l}\text { Mulheres a partir dos } 50 \text { anos e não antes dos } 40 \text { anos } \\
\text { A partir dessa idade, todos os } 2 \text { anos até aos } 70-75 \text { anos }\end{array}$ \\
\hline Colo do útero & $\begin{array}{l}\text { Tanto a citologia (Pap) como a pesquisa de virus do papilloma humano. } \\
\text { Se a citologia for usada para rastreio, início aos } 25-35 \text { anos e a partir daí cada 3-5 } \\
\text { anos. } \\
\text { Se a pesquisa de HPV for usada para rastreio, início aos } 35 \text { anos (geralmente não } \\
\text { antes dos } 30 \text { ) e a partir daí cada 3-5 anos } \\
\text { Independentemente do teste usado, as mulheres deverão participar no rastreio até } \\
\text { aos 60-65 anos, e continuar a realizá-lo, excepto se os testes mais recentes forem } \\
\text { normais. }\end{array}$ \\
\hline Colorrectal & $\begin{array}{l}\text { Homens e mulheres a partir dos 50-60 anos } \\
\text { A partir dessa idade, cada } 2 \text { anos se o teste for a pesquisa de sangue oculto nas } \\
\text { fezes (gFOBT) ou o teste imunoquímico fecal (FIT) } \\
\text { A partir dessa idade, cada } 10 \text { anos ou mais se o teste de rastreio for a } \\
\text { sigmoidoscopia flexível (FS) ou a colonoscopia (CT). } \\
\text { A maioria dos programas continua a enviar convites para rastreio até aos 70-75 anos }\end{array}$ \\
\hline
\end{tabular}

Estas recomendações baseiam-se, sobretudo, em estudos randomizados para o caso dos rastreios do cancro da mama e do cancro colorrectal, e em estudos observacionais no caso do cancro do colo do útero, em que a evidência não-experimental foi de tal modo clara que não se considerou necessário realizar estudos randomizados.

A definição do género e do grupo etário da população-alvo é importante, baseando-se nos conceitos de estratificação de risco - factores de risco, incidência, prevalência, mortalidade - e nas características operativas dos testes de rastreio - sensibilidade, especificidade e valores preditivos - de molde a permitir uma "boa" definição dos casos verdadeiro-negativos e verdadeiro-positivos, bem como a minimizar os casos falso-negativos e falso-positivos.

O intervalo de rastreio está associado à história natural da doença e aos tempos "teóricos" de aparecimento e de progressão tumoral.

\subsection{Rastreio do Cancro da Mama Feminina}

Os vários estudos realizados têm, de uma forma consistente, evidenciado a eficácia e a efectividade do rastreio de cancro da mama. Meta-análises de ensaios clínicos randomizados e revisões sistemáticas mostram uma significativa redução no risco relative da mortalidade por cancro da mama entre 0.80-0.82, apesar de diferenças no acompanhamento e no outcome. (Gotzsche \& Jorgensen, 2013; Fitzpatrick-Lewis, Hodgson \& Ciliska, 2011; Independent, 2012)

Os resultados dos estudos observacionais evidenciam também reduções significativas da mortalidade, tanto quando se consideram as idades limítrofes aos 50-69 anos, entre 25 e 48\%, tal como em estudos de tendência temporal, da ordem dos 1-9\% ao ano, e entre 28 e 36\% numa análise pré e pós-rastreio no intervalo entre 15 e 30 anos de acompanhamento. (Broeders et al., 2012; Gabe \& Duffy, 2005).

\subsection{Rastreio do Cancro do Colo do Útero}

A A evidência da efectividade do rastreio de cancro do colo do útero com citologia convencional (teste de Papanicolau) provém "apenas" de estudos de observação, seja de estudos de coorte, caso-controlo, tendência temporal e ecológicos, podendo estimar-se a redução da incidência em cerca de 66\% (Strategies 2005), com variações muito dependentes do (maior) grau de organização e da (maior) taxa de participação das populações-alvo. 
A posterior introdução alternativa do teste de HPV, seja sequencialmente ao anterior seja como teste de rastreio inicial, evidenciou resultados muito significativos comparativamente ao teste de Papanicolau. (Ronco et al., 2014; Ronco et al., 2006; Ronco et al., 2008).

Quanto às idades mais apropriadas para rastreio, os dados disponíveis sugerem que este terá um melhor custoefectividade em idades superiores aos 30 anos.

\subsection{Rastreio do Cancro Colorrectal}

Uma meta-análise de estudos randomizados (Hewitson et al., 2007) com utilização do teste de pesquisa de sangue oculto nas fezes pelo método "gFOBT" permitiu evidenciar uma redução de cerca de $16 \%$ na mortalidade por cancro colorrectal, embora sem redução da incidência. O uso do teste imunoquímico fecal (FIT) permitiu uma redução de cerca de $32 \%$ na mortalidade do segmento rectal, mas sem aparente impacto na redução na mortalidade por cancro do cólon ou conjunto do tracto colorrectal (Zheng et al., 2003).

No caso do uso de sigmoidoscopia flexível (FS), evidenciou-se uma redução da mortalidade entre 28 e $50 \%$ (Elmunzer et al., 2012; Scand, 1999; Hoff et al., 2009; Atkin et al., 2010; Segnan et al., 2011; Schoen et al., 2012). Já para o uso da colonoscopia (CT) os estudos de observação parecem indicar uma redução de 29 a $65 \%$ de redução da mortalidade por cancro colorrectal e de 48 a 67\% na incidência (Winawer et al., 1993; Baxter et al., 2009; Brenner et al., 2010; Brenner et al., 2011).

Os vários estudos já publicados parecem indicar que a idade óptima de rastreio parece estar nos 50-69 anos para qualquer dos testes utilizados, com diferentes eficácias marginais nos utentes dentro destes grupo etário e nas idades limítrofes, provavelmente porque aqules utilizam diferentes critérios tempo de seguimento e de outcome (Hardcastle et al., 1996; Kronborg et al., 1996; Lindholm, Brevinge, \& Haglind, 2008; Mandel et al., 1993; Scholefield et al., 2002; Mandel et al., 1999).

\subsection{Efeitos Indesejáveis do Rastreio}

Sendo a mortalidade o indicador primário de eficácia, eficiência e efectividade do rastreio organizado populacional, com reflexo populacional, haverá situações major que tem um potencial enorme na diminuição desse impacto (figura 1).

Na verdade, poderá haver cancro nos não-convidados e nos não-participantes, bem como poderá haver "cancros de intervalo" (cancros diagnosticados após um resultado negativo na avaliação do teste de rastreio, na avaliação no processo de aferição, no diagnóstico final - "verdadeiros" cancros de intervalo por não existirem ou, "falsosnegativos" por não serem detectados, por erro no procedimento -, e antes de nova ronda de rastreio).

Outro dos efeitos indesejáveis potenciais do rastreio é o "sobrediagnóstico" (“overdiagnosis"). Este termo assume que haverá cancros diagnosticados que nunca iriam causar sintomas ou a morte durante a esperança de vida expectável dos utentes e que, além disso, conduzem a um tratamento que não seria, de outro modo, necessário. Sendo uma estimativa (pois não há, nem eticamente pode haver estudos comparativos entre diagnosticar e tratar versus diagnosticar e não tratar), não temos números reais e, além disso, o método de cálculo tem variado muito entre os diferentes investigadores (Independent, 2012).

O eventual efeito iatrogénico de procedimentos de rastreio e de diagnóstico tem sido também alvo de estudo, sobretudo no caso das radiações no uso da mamografia e no caso de perfurações, sangramento e problemas cardiovasculares no caso da colonoscopia. No caso da mamografia tem havido estudos sobre o efeito prejudicial das radiações, sobretudo quando a sua utilização é introduzida precocemente e a mulher cumpre a grande maioria das voltas (de 2 em 2 anos). A sua quantificação é difícil e poderá ser estimada no aparecimento de 1,6 cancros por 100.000 mulheres (Gelder et al., 2011; Yaffe, \& Mainprize, 2011). Quanto à colonoscopia, as estimativas apontam para uma percentagem de 0,06 a 0,5\% (Quintero et al., 2012; Pox et al., 2012). 
Atenção especial tem sido dada, sobretudo nos últimos tempos, à morbilidade psicológica decorrente de qualquer das etapas do rastreio, com estudos de avaliação e introdução de medidas conducentes à sua diminuição (Brett et al., 2005; Bond et al., 2013; Brewer, Salz, \& Lillie, 2007). A informação é aqui um factor fundamental.

\subsection{Desafios}

Actualmente, a população possui cada vez maior acesso a fontes de informação em saúde (nem sempre as mais correctas) e tem, naturalmente, aumentado a sua literacia. Fundamentalmente, deseja maior esperança de vida e com maior qualidade. É neste quadro que crescem os desafios que a sociedade, em geral, e os cuidados de saúde, em particular, têm que solucionar.

Entre estes seriam de destacar

- a diminuição das barreiras aos rastreios,

- a atenção a novos testes passíveis de serem utilizados,

- a atenção aos estudos que, neste momento, avaliam a possível integração de outras localizações tumorais no "cabaz de cancros rastreáveis",

- o estudo de intervenções, passivas ou activas, de diminuição da morbilidade psicológica,

- o reforço da informação dos utentes,

- a formulação de critérios eficazes de estratificação de risco para rastreio de populações de alto-risco.

Serão aspectos que, forçosamente, veremos desenvolver num futuro mais ou menos distante.

\section{Referências}

Andermann, A. et al. (2008). Revisiting Wilson and Jungner in the genomic age: a review of screening criteria over the past 40 years. Bull World Health Organ (86), 317-319.

Arbyn, M., Anttila, A., Jordan, J., Ronco, G., Schenck, U., Segnan, N., et al. (2008). European Guidelines for Quality Assurance on Cervical Cancer Screening, 2nd edition, European Community, Brussels; Office for Official Publications of the European Communities, Luxembourg.

Armaroli, P., et al. (2015). European Code Against Cancer, 4th Edition: Cancer Screening. Cancer Epidemiology, 39S, S139-S152.

Atkin,W. S., Edwards, R., Kralj-Hans, I., Wooldrage, K., Hart, A. R., Northover, J. M., et al. (2010). Once-only flexible sigmoidoscopy screening in prevention of colorectal cancer: a multicentre randomised controlled trial, Lancet 375 , 1624-1633.

Baxter, N. N., Goldwasser, M. A., Paszat, L. F., Saskin, R., Urbach, D. R., Rabeneck, L. (2009). Association of colonoscopy and death from colorectal cancer, Ann. Intern. Med. 150, 1-8.

Bond, M., Pavey, T., Welch, K., Cooper, C., Garside, R., Dean, S, et al.. (2013). Systematic review of the psychological consequences of false-positive screening mammograms, Health Technol. Assess. 17, 1-170 v-vi.

Brenner, H., Chang-Claude, J., Seiler, C. M., Rickert, A., \& Hoffmeister, M. (2011). Protection from colorectal cancer after colonoscopy: a population-based, case-control study, Ann. Intern Med. 154, 22-30.

Brenner, H., Hoffmeister, M., Arndt, V., Stegmaier, C., Altenhofen, L., \& Haug, U. (2010). Protection from right-and left-sided colorectal neoplasms after colonoscopy: population-based study, J. Natl. Cancer Inst. 102, 89-95.

Brett, J., Bankhead, C., Henderson, B., Watson, E., \& Austoker, J. (2005). The psychological impact of mammographic screening. A systematic review, Psycho-Oncol. 14, 917-938.

Brewer, N. T., Salz, T., \& Lillie, S. E. (2007). Systematic review: the long-term effects of false-positive mammograms, Ann. Intern. Med. 146, 502-510. 
Broeders, M., Moss, S., Nystrom, L., Njor, S., Jonsson, H., Paap, E., et al. (2012). The impact of mammographic screening on breast cancer mortality in Europe: a review of observational studies, J. Med. Screen. 19(Suppl. 1), 1425.

Council of the European Union. (2013). Council recommendation of 2 December 2003 on cancer screening (2003/878/EC). Offical Journal of the European Union, L 327/34.

Elmunzer, B.J., Hayward, R. A., Schoenfeld, P. S., Saini, S. D., Deshpande, A., \& Waljee, A. K. (2012).

Effect of flexible sigmoidoscopy-based screening on incidence and mortality of colorectal cancer: a systematic review and meta-analysis of randomized controlled trials, PLoS Med. 9, e1001352.

European Commission. (2006). European Guidelines for Quality Assurance in Breast Cancer Screening and Diagnosis. In N. Perry, M. Broeders, C. de Wolf (Eds.), 4th edition, Luxembourg: Office for Official Publications of the European Communities.

Fitzpatrick-Lewis, D., Hodgson, N., Ciliska, D. (2011). Breast Cancer Screening, McMaster University, Hamilton, Ontario, Canada.

Gabe, R., Duffy, S. W. (2005). Evaluation of service screening mammography in practice: the impact on breast cancer mortality, Ann. Oncol. 16(Suppl. 2), 53-62.

Gelder, R., Draisma, G., Heijnsdijk, E. A., \& de Koning, H. J. (2011). Population-based mammography screening below age 50: balancing radiation-induced vs prevented breast cancer deaths, Br. J. Cancer 104, 1214-1220.

Gotzsche, P.C., \& Jorgensen, K. J. (2013). Screening for breast cancer with mammography, Cochrane Database Syst Rev. 6 CD001877.

Hakama, M. et al. 2008. Cancer screening: Evidence and practice in Europe 2008. European Journal of Cancer, 44: 1404-1413.

Hardcastle, J.D., Chamberlain, J. O., Robinson, M. H., Moss, S. M., Amar, S. S., Balfour, T. W., et al. (1996). Randomised controlled trial of faecal-occult-blood screening for colorectal cancer, Lancet 348, 1472-1477.

Hewitson, P., et al. (2007). Screening for colorectal cancer using the faecal occult blood test, Hemoccult, Cochrane Database Syst. Rev. (2007), CD001216

Hoff, G., Grotmol, T., Skovlund, E., \& Bretthauer, M. (2009). Norwegian colorectal cancer prevention study G. Risk of colorectal cancer seven years after flexible sigmoidoscopy screening: randomised controlled trial, BMJ 338, b1846.

Independent. (2012). UKPoBCS, The benefits and harms of breast cancer screening: an independente review, Lancet $380,1778-1786$.

Karsa, L. et al. 12013). European guidelines for quality assurance in colorectal cancer screening and diagnosis: Overview and introduction to the full Supplement publication. Endoscopy, 45, 51-59.

Kronborg, O., Fenger, C., Olsen, J., Jorgensen, O. D., \& Sondergaard, O. (1996). Randomised study of screening for colorectal cancer with faecal-occult-blood test, Lancet 348, 1467-1471.

Lindholm, E., Brevinge, H., \& Haglind, E. (2008). Survival benefit in a randomized clinical trial of faecal occult blood screening for colorectal cancer, Br. J. Surg. 95, 1029-1036.

Mandel, J. S., Church, T. R., Ederer, F., Bond, J. H. Colorectal cancer mortality: effectiveness of biennial screening for fecal occult blood, J. Natl. Cancer Inst. 91, 434-437, 1999

Mandel, J. S., Bond, J. H., Church, T. R., Snover, D.C., Bradley, G. M., Schuman, L. M., et al. (1993). Reducing mortality from colorectal cancer by screening for fecal occult blood: Minnesota Colon Cancer Control study, N. Engl. J. Med. 328, 1365-1371.

Quintero, E., Castells, A., Bujanda, L., Cubiella, J., Salas, D., Lanas, A., et al. (2012). Colonoscopy versus fecal immunochemical testing in colorectal-cancer screening, N. Engl. J. Med. 366, 697-706. 
Pox, C.P., Altenhofen, L., Brenner, H., Theilmeier, A., Von Stillfried, D., \& Schmiegel, W. (2012). Efficacy of a nationwide screening colonoscopy program for colorectal cancer, Gastroenterology 142, 1460-1467 e2

Ronco, G., Dillner, J., Elfstrom, K.M., Tunesi, S., Snijders, P. J., Arbyn, M., et al. (2014). Efficacy of HPV-based screening for prevention of invasive cervical cancer: follow-up of four European randomised controlled trials, Lancet $383,524-532$

Ronco, G., N. Segnan, P. Giorgi-Rossi, M. Zappa, G.P. Casadei, F. Carozzi, et al.. 2006. Human papillomavirus testing and liquid-based cytology: results at recruitment from the new Technologies for cervical cancer randomized controlled trial, J. NatL. Cancer Inst. 98, 765-774.

Ronco, G., Giorgi-Rossi, P., Carozzi, F., Confortini, M., Dalla Palma, P., Del Mistro, A., et al. (2008). Results at recruitment from a randomized controlled trial comparing human papillomavirus testing alone with conventional cytology as the primary cervical cancer screening test, J. Natl. Cancer Inst. 100, 492-501.

Scand, J. (1999). Population-based surveillance by colonoscopy: effect on the incidence of colorectal cancer: Telemark Polyp Study I. Gastroenterol. 34, 414-420.

Schoen, R. E., Pinsky, P. F., Weissfeld, J. L., Yokochi, L. A., Church, T., Laiyemo, A.O., et al. (2012). Colorectal-cancer incidence and mortality with screening flexible sigmoidoscopy, N. Engl. J. Med. 366: 2345-2357.

Scholefield, J. H., Moss, S. M., Mangham, C. M., Whynes, D. K., \& Hardcastle, J. D. (2012). Nottingham trial of faecal occult blood testing for colorectal cancer: a 20-year follow-up, Gut 61, 1036-1040.

Scholefield, J. H., Moss, S., Sufi, F., Mangham, C. M., \& Hardcastle, J. D. (2002). Effect of faecal occult blood screening on mortality from colorectal cancer: results from a randomised controlled trial, Gut 50, 840-844.

Segnan, N., Patnick, J., \& von Karsa, L. (2010). European Guidelines for Quality Assurance in Colorectal Cancer Screening and Diagnosis, 1st edition, European Commission, Publications Office of the European Union, Luxembourg.

Segnan, N., Armaroli, P., Bonelli, L., Risio, M., Sciallero, S., Zappa, M., et al.. (2011). Onceonly sigmoidoscopy in colorectal cancer screening: follow-up findings of the Italian randomized controlled trial-SCORE, J. Natl. Cancer Inst. 103, 1310-1322.

Strategies IWGotEoCP. (2005). Cervix Cancer Screening. IARC Handbooks of Cancer Prevention No. 10, IARC, Lyon.

Yaffe, M. J., Mainprize, J. G. (2011). Risk of radiation-induced breast cancer from mammographic screening, Radiology 258, 98-105.

Wilson, J. M. G., \& Jungner, G. (1968). Principles and practice of screening for disease. Geneva: WHO. Retrieved from: http://www.who.int/bulletin/volumes/86/4/07-050112BP.pdf

Winawer, S.J., Zauber, A. G., Ho, M. N., O’Brien, M. J., Gottlieb, L. S., Sternberg, S. S., et al. (1993). Prevention of colorectal cancer by colonoscopic polypectomy: The National Polyp Study Workgroup, N. Engl. J. Med. 329, 19771981.

Zheng, S., et al. (2003). Cluster randomization trial of sequence mass screening for colorectal cancer, Dis. Colon Rectum 46, 51-58.

\section{Sobre os Autores}

Vitor José Lopes Rodrigues (PhD) Actualmente é Professor Associado de Epidemiologia e Medicina Preventiva e Director do Instituto de Higiene e Medicina Social a Faculdade de Medicina da Universidade de Coimbra (Portugal). Nasceu em Coimbra, Portugal, em 1957. Licenciou-se em Medicina em 1981 e doutorou-se em Epidemiologia e Saúde Pública em 1993 pela Faculdade de Medicina da Universidade de Coimbra. Tem sido coordenador de programas de rastreio de cancro da mama desde a década de 90 , consultor de registos de cancro populacional, membro da rede europeia e internacional de rastreio de cancro e co-autor de European Guidelines em Cancro da Mama e Cancro Colorectal. É perito europeu em controlo e garantia de qualidade em cancro da mama. 